DRAFT VERSION NOVEMBER 13, 2018

Preprint typeset using LTEX style emulateapj v. 11/10/09

\title{
WATER MASERS IN THE ANDROMEDA GALAXY: THE FIRST STEP TOWARD PROPER MOTION
}

\author{
JEREMY DARLING ${ }^{1}$ \\ Draft version November 13, 2018
}

\begin{abstract}
We have detected and confirmed five water maser complexes in the Andromeda Galaxy (M31) using the Green Bank Telescope. These masers will provide the high brightness temperature point sources needed for proper motion studies of M31, enabling measurement of its full three-dimensional velocity vector and its geometric distance via proper rotation. The motion of M31 is the keystone of Local Group dynamics and a gateway to the dark matter profiles of galaxies in general. Our survey for water masers selected 206 luminous compact

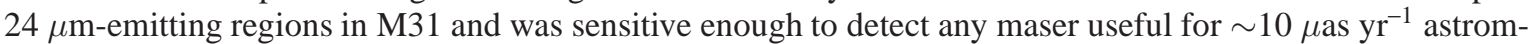
etry. The newly discovered masers span the isotropic luminosity range $0.3-1.9 \times 10^{-3} L_{\odot}$ in single spectral components and are analogous to luminous Galactic masers. The masers are distributed around the molecular ring, including locations close to the major and minor axes, which is nearly ideal for proper motion studies. We find no correlation between $24 \mu \mathrm{m}$ luminosity and water maser luminosity, suggesting that while water masers arise in star-forming regions, the nonlinear amplification pathways and beamed nature of the water masers means that they are not predictable based on IR luminosity alone. This suggests that there are additional bright masers to be found in M31. We predict that the geometric distance and systemic proper motion of M31 can be measured in 2-3 years with current facilities. A "moving cluster" observation of diverging masers as M31 approaches the Galaxy may be possible in the long term.
\end{abstract}

Subject headings: astrometry — galaxies: individual (M31) — galaxies: ISM — Local Group — masers — radio lines: galaxies

\section{INTRODUCTION}

The dominant galaxies in the Local Group, the Milky Way and Andromeda (M31), are likely to collide in the next 5-10 Gyr (Loeb et al. 2005). In the meantime, however, we would like to know the proper (transverse) motion of M31 with respect to the Milky Way. Peebles et al. (2001) show that measurements of the transverse motions of Local Group galaxies will critically test the fundamental assumption that luminosities trace the mass of galaxies. The proper motion of M31 is key to our understanding of the Local Group's future (and past) dynamical evolution as well as the density profiles and distribution of dark matter halos (Loeb et al. 2005; ; Reid et al. 2010).

Loeb et al. (2005) suggest that GAIA, SIM, or the Square

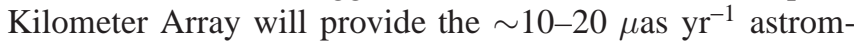
etry required for a proper motion determination of M31 (e.g., van der Marel \& Guhathakurta 2008). But Galactic analog masers in M31 observed with sensitive Very Long Baseline Interferometers (VLBI) over a time baseline of a few years would be adequate. Indeed, $22 \mathrm{GHz}$ water $\left(\mathrm{H}_{2} \mathrm{O}\right)$ masers have been discovered in M31's satellite galaxies M33 (Churchwell et al. 1977) and IC 10 (Henkel, Wouterloot, \& Bally 1986), and Brunthaler et al. (2005, 2007) have measured their proper motions, yielding their 3-dimensional velocities with respect to the Milky Way. Brunthaler et al. (2005) also measured the proper rotation of M33, which provides a geometric determination of its distance via "rotational parallax." Loeb et al. (2005) show that a backwards-time-evolution model has M33 colliding with M31 in the past. Since M33 does not bear the morphological signatures of such an interaction (but does show star forma-

\footnotetext{
${ }^{1}$ Center for Astrophysics and Space Astronomy, Department of Astrophysical and Planetary Sciences, University of Colorado, 389 UCB, Boulder, CO 80309-0389; jdarling@ origins.colorado.edu
}

tion possibly triggered by a close pass), one must assume that M31 was elsewhere at the time and thus has a non-negligible tangential motion, of order $100 \mathrm{~km} \mathrm{~s}^{-1}$ (Loeb et al. 2005; Brunthaler et al. 2007, but see van der Marel \& Guhathakurta (2008)).

Detecting masers in M31 has historically been problematic (Sullivan 1973). Greenhill et al. (1995) and Imai et al. (2001) conducted pointed $\mathrm{H}_{2} \mathrm{O}$ maser surveys toward $\mathrm{H}$ II regions in M31, reaching $1 \sigma$ rms noise levels of 29 and $70 \mathrm{mJy}$, respectively. Claussen \& Beasley (private communication) conducted a survey for $\mathrm{H}_{2} \mathrm{O}$ masers in the nuclear region and across most of the molecular ring with the Very Large Array, making no detections and reaching an rms noise of $30 \mathrm{mJy}$ per beam. Sjouwerman et al. (2010) have detected the first maser of any type in $\mathrm{M} 31$, a Class II methanol $\left(\mathrm{CH}_{3} \mathrm{OH}\right)$ maser at $6.7 \mathrm{GHz}$, but no associated water masers were detected. The water maser non-detections to date are all consistent with the aggregate star formation rate of $\mathrm{M} 31$, and these surveys were not sufficiently sensitive to have detected a typical Galactic maser at the distance of M31 (Brunthaler et al. 2006). The lack of Galactic analog water maser detections in M31 has simply been a problem of sensitivity and of locating likely sites of maser activity, both of which now have remedies.

Galactic analog $\mathrm{H}_{2} \mathrm{O}$ masers associated with star formation are detectable in M31 in reasonable integration time using the Green Bank Telescope 2 (GBT); they already have been detected in significantly more distant galaxies (Darling, Brogan, $\&$ Johnson 2008). But a full survey of the star-forming regions of M31 with the sensitivity to detect Galactic analog water masers is prohibitive because the galaxy is so large on the sky. We thus combined an order of magnitude sensitivity improvement compared to the best previous survey with

\footnotetext{
2 The National Radio Astronomy Observatory is a facility of the National Science Foundation operated under cooperative agreement by Associated Universities, Inc.
} 


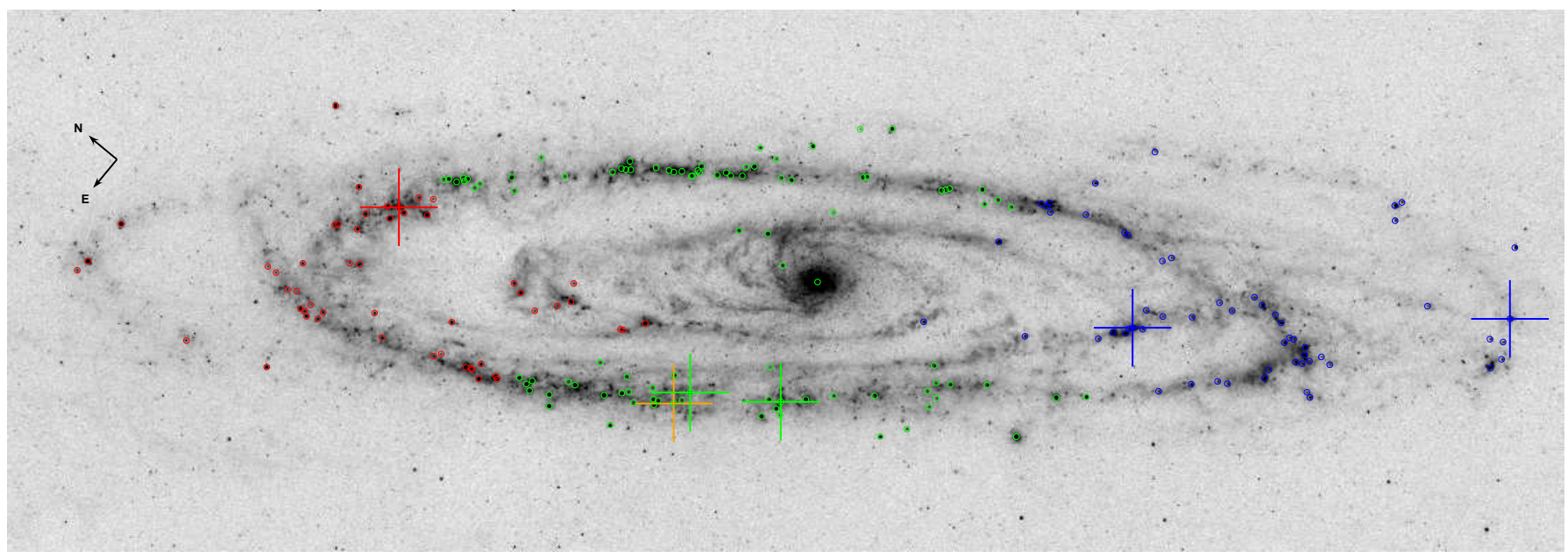

FIG. 1.- Spitzer $24 \mu \mathrm{m}$ map of M31 (Gordon et al. 2008). The circles indicate the 206 pointing centers (the circles are to scale, showing the $33^{\prime \prime}$ FWHM beam). Crosses mark the detected masers and are enlarged for clarity. The orange cross indicates the $6.7 \mathrm{GHz}$ methanol maser detected by Siouwerman et al. (2010). Colors indicate the systemic (green), red, and blue spectrometer tuning centers at $-300,-100$, and $-500 \mathrm{~km} \mathrm{~s}^{-1}$, respectively.

a physically motivated pointing guide to the most likely sites of water maser activity. In this Letter, we describe the source selection, the observations and data reduction, and the newly discovered water masers. We also discuss the prospects for using these masers for proper motion studies of M31.

Throughout this Letter, we use heliocentric velocities (optical definition) and assume the following about M31: a systemic velocity of $-300 \mathrm{~km} \mathrm{~s}^{-1}$ (de Vaucouleurs et al. 1991), a distance of $780 \mathrm{kpc}$ (McConnachie et al.|2005), a major axis position angle of $38^{\circ}(\mathrm{E}$ of $\mathrm{N})$, a constant inclination of $77^{\circ}$, and a central coordinates 00:42:44.3, +41:16:09 (J2000).

\section{SAMPLE SELECTION}

A Spitzer $24 \mu \mathrm{m}$ map of M31 (Gordon et al. 2008) guided the GBT observations in two important ways: source selection and completeness. The association of water masers with (ultra)compact H II regions is well known, but the molecular gas as traced by $\mathrm{CO}$ emission does not necessarily indicate these regions. Likewise, $\mathrm{H} \alpha$-selected $\mathrm{H}$ II regions may select against dust-enshrouded ultra-compact $\mathrm{H}$ II regions. Compact $24 \mu \mathrm{m}$ emission, however, is a good indicator of likely $\mathrm{H}_{2} \mathrm{O}$ masers, and the rough relationship between far-IR emission and water maser luminosity is well-known (e.g., Jaffe et al. 1981; Castangia et al. 2008). We therefore selected compact (unresolved at the $23.5 \mathrm{pc}$ resolution of the Spitzer map) $24 \mu \mathrm{m}$ sources associated with the dusty molecular - and presumably star-forming — regions in M31. We constructed a catalog of 206 of these objects working from the brightest down to the point where most of the $24 \mu \mathrm{m}$ emission becomes extended, about $4 \mathrm{MJy}^{\mathrm{steradian}}{ }^{-1}$ at peak specific intensity. Although this pointed survey does not include all star formation in M31, it does include a large fraction of the likely sites for strong $\mathrm{H}_{2} \mathrm{O}$ maser activity, and by working down in luminosity through the catalog of compact $24 \mu \mathrm{m}$ sources, we have surveyed a large fraction of the total ongoing star formation in the galaxy (e.g., Tabatabaei \& Berkhuijsen 2010) and thus most of the likely $\mathrm{H}_{2} \mathrm{O}$-bearing regions ( $\$ 5$. Figure 1 shows the $24 \mu \mathrm{m}$ map of M31 with the pointing centers and primary beam size for our GBT water maser survey.

\section{OBSERVATIONS AND DATA REDUCTION}

We observed the $6_{16}-5_{23} 22.23508 \mathrm{GHz}$ ortho-water maser line toward $20624 \mu \mathrm{m}$ sources in M31 with the GBT in 2010
October through December. A $670 \mathrm{~km} \mathrm{~s}^{-1}$ (50 MHz) bandpass was centered on a heliocentric velocity of $-300 \mathrm{~km} \mathrm{~s}^{-1}$ for 97 sources in the central parts of the galaxy and along the minor axis, on $-100 \mathrm{~km} \mathrm{~s}^{-1}$ for 52 sources in the redshifted northeast wedge of the galaxy, and on $-500 \mathrm{~km} \mathrm{~s}^{-1}$ for 57 sources in the blueshifted southwest wedge of the galaxy (Table 1).

Observations were conducted with the dual K-band receivers in a nodding mode in two circular polarizations with $0.16 \mathrm{~km} \mathrm{~s}^{-1}(12.2 \mathrm{kHz})$ channels and 9-level sampling. The time on-source was 5 minutes except for sources that were reobserved to confirm or refute possible lines (typically $10 \mathrm{~min}$ utes; see Table 11. A winking calibration diode and hourly atmospheric opacity estimates were used for flux density calibration. Opacities ranged from 0.05 to 0.13 nepers but were typically 0.07 nepers. The estimated uncertainty in the flux density calibration is $\sim 20 \%$. Pointing was typically good to within a few arcseconds and the largest pointing drifts during observations were no more than $6^{\prime \prime}$. The resolution of the $24 \mu \mathrm{m}$ Spitzer image is $6^{\prime \prime}$ Gordon et al. 2008), so the unresolved IR sources remained within the $33^{\prime \prime}$ GBT beam even during the largest pointing drifts. The $33^{\prime \prime}$ beam (FWHM) at $22 \mathrm{GHz}$ spans $125 \mathrm{pc}$ in M31.

After averaging polarizations, spectra were Hanning smoothed and subsequently Gaussian smoothed to obtain a final spectral resolution of $3.3 \mathrm{~km} \mathrm{~s}^{-1}(244 \mathrm{kHz})$ for the line search. Polynomial baselines, typically of fifth order, were fit and subtracted to obtain flat and generally uniform-noise spectra. Spectral rms noise measurements for each pointing center are listed in Table 1. Non-detection spectra generally did not show any features greater than $3 \sigma$, corresponding to roughly $10 \mathrm{mJy}$, at least an order of magnitude more sensitive than previous surveys (Greenhill et al. 1995; Imai et al. 2001, Claussen \& Beasley, priv. comm.) and likely the weakest line that can currently be used for VLBI proper motion studies. All data reduction was performed in GBTIDL 3 .

\section{RESULTS}

We have detected water maser emission in five out of 206 $24 \mu \mathrm{m}$-selected regions in M31 (Table 1). Most of these are

\footnotetext{
${ }^{3}$ GBTIDL (http: //gbtidl.nrao.edu//) is the data reduction package produced by NRAO and written in the IDL language for the reduction of GBT data.
} 
TABLE 1

WATER MASER SURVEy SAMPLE

\begin{tabular}{|c|c|c|c|c|c|c|c|c|c|c|c|}
\hline $\begin{array}{l}\text { Source } \\
\text { J2000 }\end{array}$ & $\begin{array}{c}V_{\text {Obs }} \\
\mathrm{km} \mathrm{s}^{-1}\end{array}$ & $\begin{array}{l}\text { rms } \\
\text { mJy }\end{array}$ & $\begin{array}{l}\text { Source } \\
\text { J2000 }\end{array}$ & $\begin{array}{c}V_{\text {Obs }} \\
\mathrm{km} \mathrm{s}^{-1}\end{array}$ & $\begin{array}{l}\mathrm{rms} \\
\mathrm{mJy}\end{array}$ & $\begin{array}{l}\text { Source } \\
\text { J2000 }\end{array}$ & $\begin{array}{c}V_{\mathrm{Obs}} \\
\mathrm{km} \mathrm{s}^{-1}\end{array}$ & $\begin{array}{l}\text { rms } \\
\text { mJy }\end{array}$ & $\begin{array}{l}\text { Source } \\
\text { J2000 }\end{array}$ & $\begin{array}{c}V_{\mathrm{Obs}} \\
\mathrm{km} \mathrm{s}^{-1}\end{array}$ & $\begin{array}{l}\text { rms } \\
\text { mJy }\end{array}$ \\
\hline $003849.2+402551.7$ & -500 & 3.2 & $003906.7+403704.5$ & -500 & 3.5 & $003910.2+403725.6$ & -500 & 3.2 & $003916.1+403629.5$ & -500 & 3.0 \\
\hline $003918.9+402158.4$ & -500 & 1.8 & $003930.2+402106.4$ & -500 & 3.7 & $003933.2+402215.6$ & -500 & 3.2 & $003937.5+402011.5$ & -500 & 3.4 \\
\hline $004031.2+403952.0$ & -500 & 2.9 & $004031.7+404127.0$ & -500 & 3.3 & $004032.6+403856.1$ & -500 & 3.3 & $004033.3+403352.1$ & -500 & 3.1 \\
\hline $004033.8+403246.6$ & -500 & 3.1 & $004034.7+403541.2$ & -500 & 2.8 & $004035.1+403701.1$ & -500 & 4.0 & $004035.8+403724.6$ & -500 & 3.6 \\
\hline $004036.1+410117.5$ & -500 & 2.1 & $004038.0+403514.9$ & -500 & 2.9 & $004038.8+403431.0$ & -500 & 3.4 & $004039.4+403730.5$ & -500 & 3.4 \\
\hline $004041.6+405105.0$ & -500 & 2.0 & $004042.1+403454.5$ & -500 & 3.4 & $004043.3+404321.9$ & -500 & 3.1 & $004043.6+403530.5$ & -500 & 3.3 \\
\hline $004058.6+404558.0$ & -500 & 2.1 & $004058.6+410332.3$ & -500 & 3.0 & $004059.8+403652.4$ & -500 & 4.8 & $004100.6+410334.0$ & -500 & 3.7 \\
\hline $004101.6+410405.8$ & -500 & 2.9 & $004102.0+410254.9$ & -500 & 1.7 & $004107.6+404812.5$ & -500 & 3.5 & $004110.4+404949.5$ & -500 & 3.1 \\
\hline $004112.5+410609.7$ & -300 & 3.4 & $004113.7+403918.6$ & -500 & 2.2 & $004113.9+410736.1$ & -300 & 2.2 & $004114.8+410923.7$ & -300 & 3.8 \\
\hline $004115.9+404011.6$ & -500 & 3.1 & $004119.1+404857.4$ & -500 & 3.6 & $004119.5+411948.8$ & -300 & 3.9 & $004120.0+410821.5$ & -300 & 3.0 \\
\hline $004121.7+404947.7$ & -500 & 2.4 & $004123.2+405000.6$ & -500 & 3.4 & $004124.8+411154.6$ & -300 & 3.4 & $004125.4+404200.4$ & -500 & 3.7 \\
\hline $004126.1+404959.1$ & -500 & 3.7 & $004126.5+411206.9$ & -300 & 3.0 & $004128.1+411222.6$ & -300 & 3.6 & $004129.8+405059.5$ & -500 & 2.2 \\
\hline $004129.8+412211.1$ & -300 & 3.1 & $004130.3+410501.7$ & -500 & 3.1 & $004137.0+405142.5$ & -500 & 3.3 & $004138.6+404401.2$ & -500 & 3.4 \\
\hline $004146.7+411846.6$ & -300 & 3.3 & $004148.2+411903.8$ & -300 & 3.6 & $004151.9+412442.1$ & -300 & 3.3 & $004159.4+405720.8$ & -500 & 2.1 \\
\hline $004241.9+405155.2$ & -300 & 3.6 & $004242.5+410001.4$ & -300 & 3.0 & $004242.5+413155.2$ & -300 & 3.9 & $004242.9+413159.8$ & -300 & 3.7 \\
\hline $004244.1+413259.2$ & -300 & 3.4 & $004244.4+411608.5$ & -300 & 3.7 & $004246.2+410111.4$ & -300 & 2. & $004247.0+413333.0$ & -300 & 4.0 \\
\hline $004247.9+413400.5$ & -300 & 3.1 & $004249.1+411945.9$ & -300 & 3.1 & $004249.3+41$ & -300 & 3.2 & $004251.0+413$ & -300 & 3.2 \\
\hline $004252.3+410014.8$ & -300 & 2.1 & $004252.4+410120.7$ & -300 & 2.8 & $004256.9+413728.1$ & -300 & 1.6 & $004258.2+410015.9$ & -300 & 3.1 \\
\hline $004300.0+413654.2$ & -300 & 2.5 & $004301.5+413717.2$ & -300 & 2.8 & $004302.5+413740.5$ & -300 & 3. & $004306.9+413807.1$ & -300 & 3.3 \\
\hline $004311.3+410459.5$ & -300 & 3.6 & $004314.2+410033.9$ & -300 & 2.4 & $004324.1+414124.7$ & -300 & 2.0 & $004324.3+414418.7$ & -300 & 1.9 \\
\hline $004324.4+410802.9$ & -300 & 2. & $4325.6+410206.4$ & -300 & 4.4 & $004333.6+411432.3$ & -300 & 2. & $004334.9+410953.6$ & -300 & 3.8 \\
\hline $004341.7+414519.4$ & -300 & 2.0 & $4343.9+411$ & -300 & 1.8 & $004346.3+414418.5$ & -300 & 3.8 & $004346.8+411239.7$ & -300 & 2.9 \\
\hline $004348.1+411133.2$ & -300 & 3.2 & $4349.4+411053.8$ & -300 & 2.4 & $004354.8+414715.6$ & -300 & 2.9 & $004355.8+411211.6$ & -300 & 4.1 \\
\hline $004356.6+412629.6$ & -100 & 2.4 & $4356.8+414831.6$ & -300 & 3.6 & $4357.7+41$ & -300 & 3. & $004358.2+414$ & -300 & 3.0 \\
\hline $004358.7+414837.5$ & -300 & 3.0 & $004401.5+414909.6$ & -300 & 3.8 & $004403.0+414954.7$ & -300 & 3.1 & $004403.9+413414.8$ & -100 & 3.4 \\
\hline $004404.9+415016.1$ & -300 & 2.8 & $004406.4+412745.0$ & -100 & 2.9 & $004407.0+41$ & -100 & 3. & $004407.8+412$ & -300 & 3.0 \\
\hline $004409.5+411856.6$ & -300 & 1.6 & $4410.5+420247.5$ & -100 & 2.9 & $004412.1+413320.5$ & -100 & 2.8 & $004415.3+411905.7$ & -300 & 2.9 \\
\hline $004416.0+414950.7$ & -100 & 3.1 & $4418.2+413406.6$ & -100 & 2.8 & $004419.2+41$ & -300 & 2. & 004 & -300 & 3.2 \\
\hline $004420.2+415$ & -100 & 3. & $.0+412$ & -300 & 3. & $004423.3+41$ & -100 & 2. & $3.7+41$ & -300 & 3.3 \\
\hline $004520.7+414716.7$ & -100 & 3.1 & $004524.4+415537.4$ & -100 & 2.1 & $004528.2+414513.6$ & -100 & 3.1 & $004536.9+415704.0$ & -100 & 3.2 \\
\hline $004537.2+415802.4$ & -100 & 2.9 & $004537.3+415107.0$ & -100 & 3.2 & 5276 & -100 & 3. & $004538.5+415$ & -100 & 3.2 \\
\hline $004540.0+415510.2$ & -100 & 3.2 & $004541.6+415107.7$ & -100 & 3.3 & $004542.9+415234.8$ & -100 & 2.5 & $004543.3+415301.1$ & -100 & 3.0 \\
\hline $004544.3+415207.4$ & -100 & 3.1 & $004608.5+421131.0$ & -100 & 2.9 & $004617.6+415158.0$ & -100 & 3.4 & $004633.6+415932.0$ & -100 & 3.0 \\
\hline $004634.4+421143.1$ & -100 & 2.4 & $004641.6+421156.2$ & -100 & 3.2 & & & & & & \\
\hline
\end{tabular}

NOTE. - 206 sources were observed for 5 minutes except for italicized sources $(10 \mathrm{~min})$. Bold sources are $\mathrm{H}_{2} \mathrm{O}$ masers; $003918+402158,004343+411137$, and $004409+411856$ were observed for 15 minutes in two sessions; $004121+404947$ was observed for 10 minutes in a single session; and $004430+415154$ was observed for 25 minutes in three sessions. No maser variability was apparent. The rms noise is for $3.3 \mathrm{~km} \mathrm{~s}^{-1}$ channels in all cases. $V_{\mathrm{Obs}}$ is the observed central velocity.

clearly maser complexes that will likely resolve into individual maser spots when imaged interferometrically. Measured line properties are listed in Table 2, spectra are plotted in Figure 2. The isotropic line luminosities of the individual maser components are analogous to luminous Galactic water masers. We also confirm the Sjouwerman et al. (2010) non-detection of water at the location of the methanol maser (Figure 1).

Wherever there is coverage, the water lines correspond to the range of $\mathrm{CO}(1-0)$ line velocities within the GBT beam (Nieten et al. 2006). The notable exceptions are $004409+411856$, in which the second water maser component does not overlap with the observed $\mathrm{CO}$ line range, and $003918+402158$ where there is no CO line coverage. In the latter, the observed maser velocities are broadly consistent with the $\mathrm{H}$ I rotation curve and its uncertainties (Chemin et al. 2009, although see $\$ 5.2$.

\section{DISCUSSION}

The GBT survey has produced some surprises: (1) the strongest water maser complex lies outside of the main starforming molecular ring in M31, and (2) while the $24 \mu \mathrm{m}$ selection method is a success, the $24 \mu \mathrm{m}$ luminosity is not predictive of maser presence or strength. Our survey has re- 
TABLE 2

WATER MASER Line Properties

\begin{tabular}{|c|c|c|c|c|c|c|}
\hline Source & Line & $\begin{array}{c}V_{\mathrm{H}_{2} \mathrm{O}} \\
\left(\mathrm{km} \mathrm{s}^{-1}\right)\end{array}$ & $\begin{array}{l}S_{\text {Peak }} \\
(\mathrm{mJy})\end{array}$ & $\begin{array}{c}\Delta V_{\mathrm{FWHM}} \\
\left(\mathrm{km} \mathrm{s}^{-1}\right)\end{array}$ & $\underset{\left(\mathrm{mJy} \mathrm{km} \mathrm{s}^{-1}\right)}{\int S \mathrm{~d} v}$ & $\begin{array}{l}L_{\mathrm{H}_{2} \mathrm{O}} \\
\left(L_{\odot}\right)\end{array}$ \\
\hline \multirow[t]{7}{*}{$003918+402158$} & 1 & $-574.30(0.07)$ & $33.5(4.7)$ & $1.0(0.2)$ & $37(8)$ & $0.00052(11)$ \\
\hline & 2 & $-566.79(0.04)$ & $61.7(4.2)$ & $1.3(0.1)$ & $87(9)$ & $0.0012(1)$ \\
\hline & 3 & $-563.59(0.08)$ & $26.9(5.8)$ & $0.8(0.2)$ & $23(8)$ & $0.00032(11)$ \\
\hline & 4 & $-561.84(0.02)$ & 122.2(9.1) & $1.13(0.09)$ & $135(15)$ & $0.0019(2)$ \\
\hline & 5 & $-559.7(0.4)$ & $26.0(2.8)$ & $3.6(1.3)$ & $99(36)$ & $0.0014(5)$ \\
\hline & 6 & $-557.63(0.04)$ & $68.5(7.1)$ & $0.9(0.1)$ & $66(11)$ & $0.00092(15)$ \\
\hline & Sum & & & & $447(43)$ & $0.0063(6)$ \\
\hline $004121+404947$ & 1 & $-528.9(0.4)$ & $17.7(2.4)$ & $5.1(0.8)$ & $95(20)$ & $0.0013(3)$ \\
\hline \multirow[t]{3}{*}{$004343+411137$} & 1 & $-297.15(0.10)$ & $21.1(4.1)$ & $1.0(0.2)$ & $23(7)$ & $0.00032(10)$ \\
\hline & 2 & $-294.01(0.04)$ & $44.9(4.8)$ & $0.74(0.09)$ & $35(6)$ & $0.00049(8)$ \\
\hline & Sum & & & & $58(9)$ & $0.00081(13)$ \\
\hline \multirow{3}{*}{$004409+411856$} & 1 & $-245.09(0.07)$ & $32.7(4.5)$ & $1.1(0.2)$ & $38(8)$ & $0.00053(11)$ \\
\hline & 2 & $-237.4(0.2)$ & $18.1(3.1)$ & $2.3(0.5)$ & $44(12)$ & $0.00062(17)$ \\
\hline & Sum & & & & $82(14)$ & $0.0011(2)$ \\
\hline $004430+415154$ & 1 & $-117.5(0.5)$ & $9.3(1.4)$ & $6.8(1.2)$ & $67(15)$ & $0.0009(2)$ \\
\hline
\end{tabular}

NотE. - Line properties determined from Gaussian fits. Isotropic line luminosities were computed from $L_{\mathrm{H}_{2} O}=\left(0.023 L_{\odot}\right) D^{2} \int S \mathrm{~d} v$, where $D$ is in $\mathrm{Mpc}$ and $\int S \mathrm{~d} v$ is in $\mathrm{Jy} \mathrm{km}$ $\mathrm{s}^{-1}$ Henkel et al. 2005). Parenthetical values indicate $1 \sigma$ statistical uncertainties.
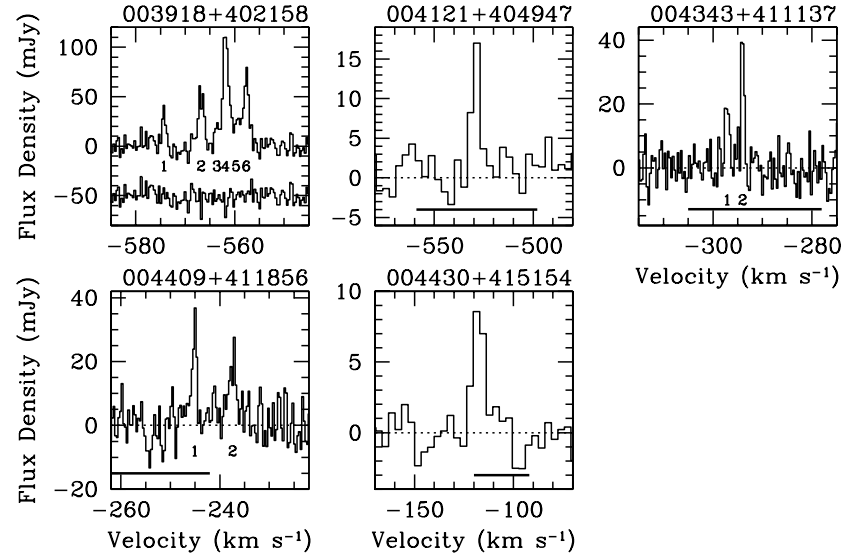

Velocity $\left(\mathrm{km} \mathrm{s}^{-1}\right)$

FIG. 2.- Spectra of the five water maser complexes detected in M31. While all of our spectra were smoothed to $3.3 \mathrm{~km} \mathrm{~s}^{-1}$ for the search, the lines of $003918+402158,004343+411137$, and $004409+411856$ are very strong and narrow and we present their spectra with $0.33 \mathrm{~km} \mathrm{~s}^{-1}$ channels. For $003918+402158$, we plot fit residuals offset by $-50 \mathrm{mJy}$. Dotted lines on the other spectra indicate the zero point. Numbers below the spectra label the lines enumerated in Table 2 The bold horizontal bars indicate the velocity range of CO within the GBT beam (Nieten et al. 2006).

vealed no correlation between $24 \mu \mathrm{m}$ luminosity and water maser luminosity, suggesting that while water masers arise in star-forming regions, the nonlinear amplification pathways and beamed nature of the water masers means that they are not predictable based on IR luminosity alone. We therefore believe that additional strong water masers may be found in M31 because our survey of $24 \mu \mathrm{m}$-emitting regions has not been exhaustive - it has simply selected the most IR-luminous compact sources (Figure 1).

It is also certain that not all of the selected $24 \mu \mathrm{m}$ sources are associated compact H II regions: cool stellar atmospheres, supernova remnants, planetary nebulae, and background AGN and galaxies can all be sources of $24 \mu \mathrm{m}$ emission (e.g., Verley et al.2007; Mould et al. 2008). Our detection statistics among the observed sample are thus not physically meaningful, although the majority of the $24 \mu \mathrm{m}$ emitters in the molecular ring of M31 are likely to be compact H II regions.

\subsection{Detection Rate and Star Formation}

The sensitivity of the GBT survey corresponds to a $~ 200 \mathrm{Jy}$ Galactic maser $6 \mathrm{kpc}$ distant. There are $23 \mathrm{H}_{2} \mathrm{O}$ masers in the Palagi et al. (1993) sample and about 30 masers in the Breen et al. (2010) sample that our survey could detect at the distance of M31. Hence, sensitivity is not the only consideration; the number of water masers detectable in M31 will also depend on the the substantially lower star formation rate of M31 compared to Galactic.

To estimate the number of expected water masers in Local Group galaxies, Brunthaler et al. (2006) used the Greenhill et al. (1990) Galactic water maser luminosity function (LF) scaled from the Galactic star formation rate to that of Local Group galaxies, assuming that maser population is proportional to the star formation rate. Local Group water maser statistics agree fairly well with this approach (Brunthaler et al. 2006). We scale the Galactic star formation rate $\left(\sim 4 M_{\odot} \mathrm{yr}^{-1}\right.$; Diehl et al. 2006) to that of $\mathrm{M} 31\left(\sim 0.35 M_{\odot} \mathrm{yr}^{-1}\right.$; Walterbos \& Braun 1994, Tabatabaei \& Berkhuijsen 2010) to predict 2.3 water masers for an isotropic line luminosity limit of $4 \times 10^{-4} L_{\odot}$, which is a $3 \sigma$ line flux limit of $9 \mathrm{mJy}$ and width $3.3 \mathrm{~km} \mathrm{~s}^{-1}$. This small number suffers from small number statistics, but our five detections suggest that either we have detected the majority of bright water masers in M31 or that the star formation scaling approach is inaccurate. This program is the first to detect the tail of the $\mathrm{H}_{2} \mathrm{O}$ maser LF in M31.

\subsection{Velocities}

A comparison of the maser velocities to the rotation of M31 is of special interest for a geometric distance determination. Proper rotation is a measure of angular frequency, and if this angular motion corresponds directly to the rotation velocity, then a geometric distance may be obtained: $D=V_{\text {Rot }} / \mu_{\text {Rot }}$. A proper rotation distance determination relies on the the masers rotating with the galaxy, but they could also exhibit their own peculiar proper motions, so a large ensemble of water masers would be ideal to reduce this source of error.

In Figure 3 we examine the correspondence between the maser velocities and the projected and inclined $\mathrm{H}$ I rotation curve (Chemin et al. 2009). Since the maser velocities are extremely well-determined (sub-km s${ }^{-1}$ ), all of the uncertainty in the velocity comparison arises from the innate velocity dispersion of the disk, the statistical uncertainty in the rotation 

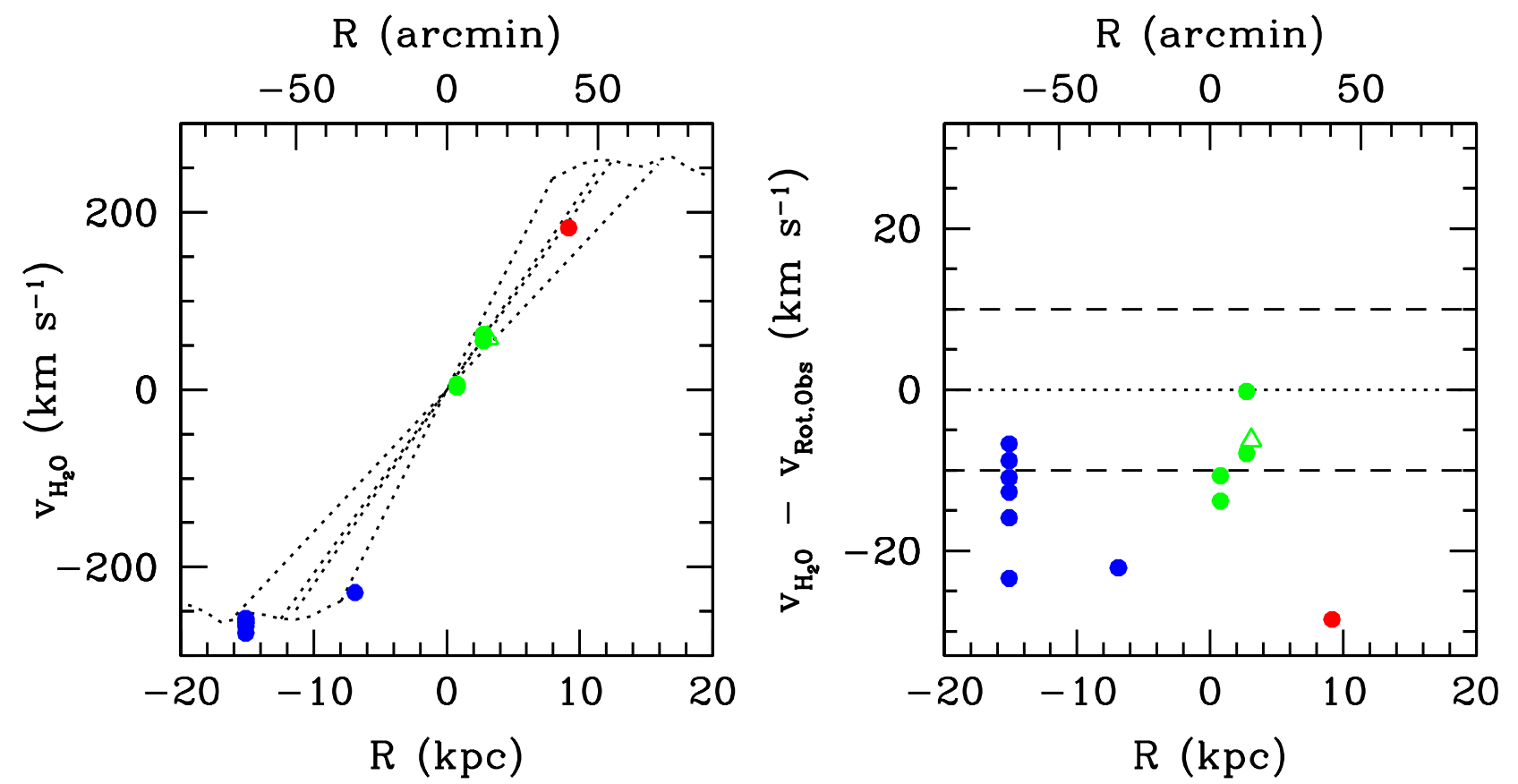

FIG. 3.- Comparison of maser velocities to the H I rotation curve of M31 (Chemin et al. 2009). Colors match those in Figure 1 except the methanol maser is indicated by a green triangle. Left: Observed water maser velocity versus position along the major axis. The dotted lines indicate the expected observed $\mathrm{H} I$ rotation curves at the radius of each maser. Once the radius is reached, the inclined rotation curve is seen along the major axis. Right: The difference between the observed water maser velocities and the projected inclined $\mathrm{H}$ I rotation velocity versus position along the major axis. The dashed lines indicate a fiducial rotation uncertainty of $\pm 10 \mathrm{~km} \mathrm{~s}^{-1}$, which is typical of M31 H I rotation curve measurements. The maser velocity uncertainties are much less than $1 \mathrm{~km} \mathrm{~s}{ }^{-1}$ (Table 22.

curve $\left(\sim 10 \mathrm{~km} \mathrm{~s}^{-1}\right)$, and the systematic uncertainties such as inclination, which can induce a rotation curve offset of order 10-15 $\mathrm{km} \mathrm{s}^{-1}$ (Corbelli et al. 2010). While the masers show fairly good agreement with the $\mathrm{H}$ I rotation, there is a notable overall blueshift that may be indicative of preferentially oriented maser amplification pathways associated with outflows. But given the propensity for masers to show peculiar velocities, a larger sample is needed to rigorously examine this effect.

\subsection{Proper Motions}

The three main contributions to the proper motion of masers in M31 are (1) the rotation of the spiral disk, (2) the systemic proper motion of M31 itself, and (3) the relative motion of masers omitting the galaxy's rotation:

(1) The direct observation of the rotation of the M31 spiral disk is the (in)famous van Maanen experiment van Maanen 1923, 1935). A rotating disk seen edge-on will show no proper motion at the tangent points and the full circular velocity along the minor axis. M31's inclined disk has a circular velocity at the molecular ring radius of about $250 \mathrm{~km} \mathrm{~s}^{-1}$, which becomes a transverse motion of $56 \mathrm{~km} \mathrm{~s}^{-1}$ at the tangent points or 15 microarcseconds ( $\mu \mathrm{as})$ year $^{-1}$. The transverse motion along the minor axis is expected to be about

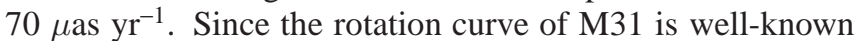
(e.g., Chemin et al. 2009; Corbelli et al. 2010), the measurement of the proper motions of masers distributed around the disk will provide a geometric distance to the galaxy, limited roughly equally by the uncertainty in the rotation model and in the proper motion measurements.

(2) The transverse motion of M31 provides the two unknown components of its three-dimensional velocity. The expected transverse velocity of M31 is of order $100 \mathrm{~km} \mathrm{~s}^{-1}$ (Loeb et al. 2005; Brunthaler et al. 2007, but see van der
Marel \& Guhathakurta 2008), or $27 \mu \mathrm{as} \mathrm{yr}^{-1}$, and measurable over two or three years (see below).

(3) There may be relative motions of masers within M31 due to peculiar deviations from the bulk rotation of the disk and due to the line of sight motion of M31 itself. The former peculiar motions are of order 10-20 $\mathrm{km} \mathrm{s}^{-1}$ (Figure 3), which is significantly smaller than the velocities discussed above and of the same order as the uncertainty in the rotation curve. These will tend to average out over an ensemble of maser complexes. The latter is the moving cluster effect caused by M31's radial velocity toward the Galaxy; M31 gets areally larger as it nears, causing relative maser separations to

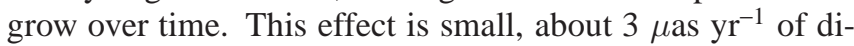
vergence for the maser pair with the largest separation, $1.79^{\circ}$. The size of this effect is equivalent to $\sim 10 \mathrm{~km} \mathrm{~s}^{-1}$, similar to maser peculiar motions. It is possible that with long time baselines and a rich network of masers one could extract the distinct signal of diverging maser spots and thus estimate the distance to M31 (but with lower precision than the proper rotation method).

How long will it take to obtain proper motions, and what are the limits on the proper motions that can be measured? The Brunthaler et al. (2005) study of the proper motion of water masers in M33 informs our expectations for M31. Water masers appear in many-component complexes that can show dramatic variability, including the appearance of new masers and the disappearance of others. But over the span of about three years, Brunthaler et al. (2005) were able to track several persistent maser features and obtain proper motions relative to background compact radio sources. Their astrometric uncertainties were on average 8-10 $\mu$ as in each epoch. Proper

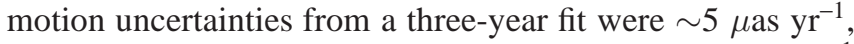
which would correspond to a transverse velocity of $18 \mathrm{~km} \mathrm{~s}^{-1}$ in M31. 
We estimate that in two 12-hour observations with the High Sensitivity Array one can obtain $1 \mathrm{mJy} r \mathrm{rms}$ noise in $1 \mathrm{~km} \mathrm{~s}^{-1}$ channels. This translates into a $6 \mu$ as uncertainty per epoch for a $40 \mathrm{mJy}$ line. In a single year we expect a $10 \sigma$ detection of the proper rotation along the minor axis, a $2.5 \sigma$ detection along the major axis, and a $4.5 \sigma$ detection of the systemic transverse proper motion of M31 (assuming $100 \mathrm{~km} \mathrm{~s}^{-1}$ ). Note that proper motion measurement errors improve as $t^{3 / 2}$ (Reid et al. 2010), so a 2-3 year astrometry campaign should be able to determine with high significance both the geometric distance to M31 and its transverse velocity.

\section{CONCLUSIONS}

We have identified the first $\mathrm{H}_{2} \mathrm{O}$ masers in the $\mathrm{M} 31$ based on pointed observations of $24 \mu \mathrm{m}$-selected regions. The $24 \mu \mathrm{m}$ selection method clearly succeeds in identifying water masers, but the $24 \mu \mathrm{m}$ properties are not predictive of maser presence or brightness. We suggest that the catalog of water masers in M31 is incomplete and that an exhaustive survey of
IR-luminous regions will identify additional masers.

The newly identified maser complexes are suitable for proper motion studies of M31. The next steps for proper motion and geometric distance measurements are interferometric localization and mapping followed by VLBI mapping and monitoring. We predict that proper rotation and systemic proper motion will be measurable in a few years with current facilities, and that the "moving cluster" divergence of maser spots as M31 approaches the Galaxy may be detectable in the long term.

The author thanks K. Gordon for the Spitzer map, M. Claussen and T. Beasley for sharing their results, K. Willett for proposal feedback, and the anonymous referee. This research has made use of the NASA/IPAC Extragalactic Database (NED) and uses observations made with the Spitzer Space Telescope, both of which are operated by the Jet Propulsion Laboratory, California Institute of Technology, under a contract with NASA.

Facilities: GBT ()

\section{REFERENCES}

Breen, S. L., Caswell, J. L., Ellingsen, S. P., \& Phillips, C. J. 2010, MNRAS, 406, 1487

Brunthaler, A., Reid, M.J., Falcke, H., Greenhill, M.J., \& Henkel, C. 2005 , Science, 307, 1440

Brunthaler, A., Henkel, C., de Blok, W. J. G., Reid, M. J., Greenhill, L. J., \& Falcke, $\mathrm{H}$.

2006, A\&A, 457, 109

Brunthaler, A., Reid, M. J., Falcke, H., Henkel, C., \& Menten, K. M. 2007, A\&A, 462, 101

Castangia, P., Tarchi, A., Henkel, C., \& Menten, K. M. 2008, A\&A, 479, 111

Chemin, L., Carignan, C., \& Foster, T. 2009, ApJ, 705, 1395

Churchwell, E., Witzel, A., Huchtmeier, W., Pauliny-Toth, I., Roland, J., \& Sieber, W. 1977, A\&A, 54, 969

Corbelli, E., Lorenzoni, S., Walterbos, R., Braun, R., \& Thilker, D. 2010, A\&A, 511, A89

Darling, J., Brogan, C., \& Johnson, K. 2008, ApJ, 685, L39

de Vaucouleurs, G., de Vaucouleurs, A., Corwin, H. G., Jr., Buta, R. J., Paturel, G., \& Fouqué, P. 1991, Third Reference Catalogue of Bright Galaxies (New York: Springer)

Diehl, R., et al. 2006, Nature, 439, 45

Gordon, K. D., et al. 2008, ApJ, 638, L87

Greenhill, L. J., et al. 1990, ApJ, 364, 513

Greenhill, L. J., Henkel, C., Becker, R., Wilson, T. L., \& Wouterloot, J. G. A. 1995, A\&A, 304, 21

Henkel, C., Wouterloot, J. G. A., \& Bally, J. 1986, A\&A, 155, 193

Henkel, C., Peck, A. B., Tarchi, A., Nagar, N. M., Braatz, J. A., Castangia, P., \& Moscadelli, L. 2005, A\&A, 436, 75
Imai, H., Ishihara, Y., Kameya, O., \& Nakai, N. 2001, PASJ, 53, 489

Jaffe, D. T., Guesten, R., \& Downes, D. 1981, ApJ, 250, 621

Loeb, A., Reid, M. J., Brunthaler, A., \& Falcke, H. 2005, ApJ, 633, 894

McConnachie, A. W., Irwin, M. J., Ferguson, A. M. N., Ibata, R. A., Lewis, G. F., \& Tanvir, N. 2005, MNRAS, 356, 979

Mould, J., Barmby, P., Gordon, K., Willner, S. P., Ashby, M. L. N., Gehrz, R. D., Humphreys, R., \& Woodward, C. E. 2008, ApJ, 687, 230

Nieten, C., Neininger, N., Guélin, M., Ungerechts, H., Lucas, R., Berkhuijsen, E. M., Beck, R., \& Wielebinski, R. 2006, A\&A, 453, 459

Palagi, F., Cesaroni, R., Comoretto, G., Felli, M., \& Natale, V. 1993, A\&A, 101,15

Peebles, P.J.E., Phelps, S.D., Shaya, E.J., \& Tully, R.B. 2001, ApJ, 554, 104 Reid, M. J., Brunthaler, A., Menten, K. M., Loinard, L., \& Wrobel, J. 2009, Astro2010, 2010, 243

Sjouwerman, L. O., Murray, C. E., Pihlstrom, Y. M., Fish, V. L., \& Araya, E. D. 2010, ApJ, 724, L158

Sullivan, W. T., III. 1973, ApJS, 25, 393

Tabatabaei, F. S. \& Berkhuijsen, E. M. 2010, A\&A, 517, 77

Unwin, S. C. 1983, MNRAS, 205, 787

van der Marel, R. P. \& Guhathakurta, P. 2008, ApJ, 678, 187

van Maanen, A. 1923, ApJ, 57, 264

van Maanen, A. 1935, ApJ, 81, 336

Verley, S., Hunt, L. K., Corbelli, E., \& Giovanardi, C. 2007, A\&A, 476, 1161

Walterbos, R. A. M., \& Braun, R. 1994, ApJ, 431, 156 